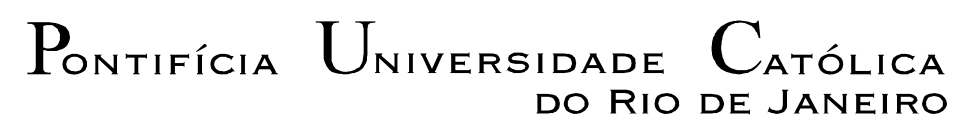

DO RIO DE JANEIRO

Pedro Paulo Suzano Xavier

Efeitos de Diferentes Regiões Urbanas na Propagação de Sinais de Sistemas de Comunicações Pessoais Baseados em Redes de Satélites Não Geoestacionários

Dissertação de Mestrado

Dissertação apresentada como requisito parcial para obtenção do título de Mestre pelo Programa de PósGraduação em Engenharia Elétrica da PUC-Rio.

Orientador: Prof. Dr. Emanoel Paiva de Oliveira Costa 


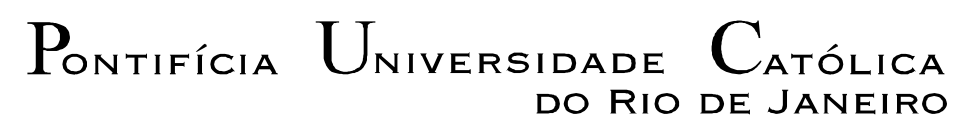

DO RIO DE JANEIRO

Pedro Paulo Suzano Xavier

\section{Efeitos de Diferentes Regiões Urbanas na Propagação de Sinais de Sistemas de Comunicações Pessoais Baseados em Redes de Satélites Não Geoestacionários}

Dissertação apresentada como requisito parcial para obtenção do grau de Mestre pelo Programa de Pós-Graduação em Engenharia Elétrica do Departamento de Engenharia Elétrica do Centro Técnico Científico da PUC-Rio. Aprovada pela Comissão Examinadora abaixo assinada.

Prof. Dr. Emanoel Paiva de Oliveira Costa Orientador Centro de Estudos em Telecomunicações - PUC-Rio

Prof. Dr. Waldecir João Perrella

Prof. Dr. Julio César Rodrigues Dal Bello

UFF

Prof. Dr. Flávio José Vieira Hasselmann Centro de Estudos em Telecomunicações - PUC-Rio

Prof. José Eugenio Leal Coordenador Setorial do Centro Técnico Científico - PUC-Rio 
Todos os direitos reservados. É proibida a reprodução total ou parcial do trabalho sem autorização da universidade, do autor e do orientador.

\section{Pedro Paulo Suzano Xavier}

Pedro Paulo Suzano Xavier graduou-se em Engenharia de Telecomunicações pela Universidade Federal Fluminense (UFF) em 2002. Ingressou no Programa de PósGraduação em Engenharia Elétrica para iniciar o curso de mestrado também em 2002, vindo a concluir o referido curso em 2005. Desde 2004 atua como professor no Centro Federal de Educação Tecnológia Celso Suckow da Fonseca (CEFET/RJ) no curso técnico em telecomunicações.

Ficha Catalográfica

Xavier, Pedro Paulo Suzano
Efeitos de diferentes regiões urbanas na
propagação de sinais de sistemas de comunicações
pessoais baseados em redes de satélites não
geostacionários / Pedro Paulo Suzano Xavier ; orientador:
Emanoel Paiva de Oliveira Costa. - Rio de Janeiro : PUC,
Departamento de Engenharia Elétrica, 2005.
106 f. ; $30 \mathrm{~cm}$
Dissertação (mestrado) - Pontifícia Universidade
Católica do Rio de Janeiro, Departamento de Engenharia
Elétrica.
Inclui referências bibliográficas.
1. Engenharia elétrica - Teses. 2. Simulação em
computadores. 3. Propagação de sinais rádio-elétricos. 4.
Regiões urbanas. 5. Sistemas de comunicações pessoais
baseados em rede. I. Costa, Emanoel Paiva de Oliveira. II.
Pontifícia Universidade Católica do Rio de Janeiro.
Departamento de Engenharia Elétrica. III. Título.




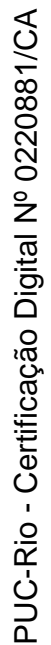

A meus pais Miguel e Angela e a meus avós Pedro Paulo e Amália. 


\section{Agradecimentos}

Ao bom DEUS por mais esta conquista.

A meus pais Miguel e Angela pela paciência, apoio, palavras de incentivo, carinho e muito amor, itens essenciais para esta conquista.

Ao meu orientador e também amigo, Prof. Emanoel Paiva de Oliveira Costa, pela atenção, apoio financeiro, respeito, consideração, paciência, fé em minha capacidade e muito mais, tendo tornado-se mais que um professor para mim.

A Sra. Maria Lúcia de Castro Junqueira, bibliotecária do CETUC e também uma inestimável amiga, pelas inúmeras horas de boas conversas e muita ajuda.

Aos amigos José Antônio Godinho Paiva, Luciana Ferreira da Silva, Pedro Castellanos, Luiz Ramirez, Sandro Szang, Rafael Ribeiro e Roque Ciufo pela amizade e companheirismo de todas as horas.

Agradeço aos meus irmãos Alessandra, Angelo e Natália pela paciência e respeito ao meu trabalho.

Um agradecimento especial aos meus avós Pedro Paulo e Amália pelo carinho e incentivo nesta jornada.

A Vice-Reitoria acadêmica pela bolsa de estudos e demais apoios institucionais sem o qual esta etapa não seria possível. 


\section{Resumo}

Xavier, Pedro Paulo Suzano. Efeitos de Diferentes Regiões Urbanas na Propagação de Sinais de Sistemas de Comunicações Pessoais Basedos em Redes de Satélites Não Geoestacionários. Rio de Janeiro, RJ, 2005. 106p. Dissertação de Mestrado - Departamento de Engenharia Elétrica, Pontifícia Universidade Católica do Rio de Janeiro.

À medida que os sistemas de comunicações móveis e de rádiodeterminação que utilizam satélites não geo-estacionários de órbitas baixas e médias ganham importância no cenário mundial de comunicações, torna-se cada vez mais necessário o conhecimento do ambiente de rádio-propagação, seu comportamento e a inter-relação entre os seus diversos aspectos e parâmetros. Em função de características dos dispositivos de comunicações utilizados em tais sistemas, como transmissores de baixa potência e antenas de baixo ou médio ganho, esses sistemas operam com margens de desvanecimento relativamente baixas. A literatura descreve uma técnica de simulação para estimação de margens de desvanecimento em diversos tipos de ambiente baseada em fotografias obtidas com a utilização de câmaras providas de lentes grande-angulares. $\mathrm{Na}$ presente dissertação, apresenta-se uma variante da metodologia original, onde se propõe a substituição da base de dados original por mapas digitais da topografia, combinada com bases de dados de edificações e de um modelo para a vegetação. Apresenta-se, ainda, a estrutura da simulação computacional utilizada para processar e analisar tais arquivos, os dados de entrada da simulação e um módulo que representa a dinâmica orbital, utilizado para prever as posições dos satélites. Finalmente, apresentam-se e analisam-se os resultados obtidos com a presente simulação. Analisa-se, também, a influência da variação dos diversos fatores que compõem os dados de entrada da simulação nos resultados obtidos.

\section{Palavras-chave}

Simulação em Computadores; Propagação de Sinais Rádio-elétricos; Regiões Urbanas; Sistemas de comunicações pessoais baseados em rede. 


\section{Abstract}

Xavier, Pedro Paulo Suzano. Effects of Different Urban Environments on the Propagation of Signals from Personal Communication Systems Based on Non Geostationary Orbit Satellite Networks. Rio de Janeiro, RJ, 2005. 106p. Master Dissertation - Department of Electrical Engineering, Pontifícia Universidade Católica do Rio de Janeiro.

As land-mobile satellite systems and radio-determination systems get more importance in the global telecommunications scenario, it becomes even more necessary to understand the radio-propagation environment: its behavior and the inter-relationship between its several aspects and physical parameters.

Due to the communications devices characteristics used in these systems, such as low-power transmitters and low/medium gain antennas, such systems operate with relatively low fade margins. The technical literature describes a simulation technique to estimate fade margins in various types of environments, based on the processing of photographs acquired by digital cameras utilizing fisheye lenses. In this dissertation, an alternate methodology is presented. It is proposed that the original database of photographs be substituted by digital elevation models combined with building databases and a vegetation model. The computer simulation structure used to process and analyzes these databases, the input simulation data, and an orbital dynamics model used to update the satellite positions are also described. Finally, the obtained simulation results are presented and analyzed. It also discusses the influence of several factors which characterize different urban regions and non geostationary orbit satellite networks in the obtained results.

\section{Keywords}

Computer simulation; Radio wave propagation; Urban environments; Personal Communication Systems (PCS); Non geostationary orbit satellite networks. 


\section{Sumário}

1 Introdução:

2 Aspectos Teóricos 19

2.1. Introdução: 19

2.2. A metodologia de Akturan e Vogel: $\quad 19$

2.3. Obtenção e Tratamento de Imagens: 20

2.4. Predição da Distribuição Cumulativa de Desvanecimento para Satélite Único: $\quad 22$

2.5. Combinação de Estados de Percursos: 25

2.6. Operação em Diversidade: 26

2.6.1. Combinação Coerente: 26

2.6.2. Diversidade por Comutação: 27

3 Nova Abordagem para Simulação 29

3.1. Introdução: $\quad 29$

3.2. Estrutura da simulação: 30

3.3. Bases de Dados de Edificações: 31

3.3.1. Formato de Apresentação dos Arquivos do IPP: 31

3.3.2. Manipulação dos Dados de Edificações: 32

3.4. Dinâmica Orbital: 36

3.4.1. Órbitas Elípticas: $\quad 37$

3.4.2. Órbitas Circulares: $\quad 39$

3.5. Estatísticas de Desvanecimento e de Eventos: 40

3.5.1. Definição dos Processos Aleatórios de Eventos e Inter-Eventos: $\quad 41$

4 Análise de Resultados Numéricos $\quad 45$

4.1. Bases de Dados de Edificações e Número de Observadores: 45

4.2. Constelações de Satélites: 48

4.3. Estatísticas de Disponibilidade: 49

4.3.1. Combinação de Estados de Percursos: 49

4.3.2. Influência da Distribuição de Alturas de Prédios e da Combinação de

Estados: $\quad 51$ 
4.4. Estatísticas de Número de Eventos de Desvanecimento: 60

4.5. Estatísticas de Duração de Eventos de Desvanecimento: 71

4.6. Estatísticas de Número de Inter-Eventos de Desvanecimento: 81

4.7. Estatísticas de Duração de Inter-Eventos de Desvanecimento: 90

4.8. Análise Comparativa das Distribuições de Número e Duração de Eventos: 99

4.9. Análise Comparativa das Distribuições de Número e Duração de Inter-

Eventos: $\quad 100$

5 Conclusões 102

6 Referências Bibliográficas 105 


\section{Lista de figuras}

Figura 2-1 - Fotografia obtida por câmara fotográfica dotada de lente grande angular para processamento posterior (reproduzida da referência [1]). 21

Figura 2-2 - Etapas de conversão e classificação de imagens na metodologia original (reproduzida da referência [1]).

Figura 3-1 - Exemplo de base de dados apresentada pelo IPP, sob o formato de arquivo de intercâmbio ".DXF" [8].

Figura 3-2 - Edificações (polígonos cinzas), meios-fios (polígonos vermelhos) e observadores (símbolos verdes) correspondentes à região de Ipanema. 33

Figura 3-3 - Modelo de concepção do objeto árvore, utilizado nas simulações em estudo, com descrição dos seus parâmetros constituintes e suas respectivas dimensões.

Figura 3-4 - Exemplo de posicionamento relativo árvore - observador, com descrição dos parâmetros de posicionamento dos mesmos.

Figura 3-5 - Imagem correspondente a um observador localizado na região de Ipanema, com os estados C, S e B apresentados nas cores azul, verde e vermelha, respectivamente.

Figura 3-8 - Definição dos processos aleatórios e sua correspondência em termos de número de eventos e duração de eventos (reproduzido da referência [14]).

Figura 3-9 - Definição da duração de um dado desvanecimento, em um dado limiar S, detalhando-se as variações positivas e negativas, indicando-se o início e o fim do evento, respectivamente (reproduzido da referência [14]).42

Figura 3-10 - Definição da variável aleatória de inter-eventos, onde I e II são casos de inter-eventos (adaptado da referência [14]).

Figura 4-2 - Comparação entre as constelações: (a) Globalstar, (b) ICO e (c) Iridium; Levando-se em consideração todas as regiões em estudo. $\quad 50$

Figura 4-3 - Comparação, para a região de Copacabana ( $1^{\circ}$ gráfico), entre as constelações Globalstar, ICO e Iridium $\left(2^{\circ}, 3^{\circ}\right.$ e $4^{\circ}$ gráficos, respectivamente e do alto para baixo).

Figura 4-4 - Comparação, para a região de Irajá ( $1^{\circ}$ gráfico), entre as constelações Globalstar, ICO e Iridium $\left(2^{\circ}\right.$, $3^{\circ}$ e $4^{\circ}$ gráficos, respectivamente e do alto para baixo). 
Figura 4-5 - Comparação, para a região de Ipanema ( $1^{\circ}$ gráfico), entre as constelações Globalstar, ICO e Iridium $\left(2^{\circ}, 3^{\circ}\right.$ e $4^{\circ}$ gráficos, respectivamente e do alto para baixo).

Figura 4-6 - Comparação, para a região de Tijuca ( $1^{\circ}$ gráfico), entre as constelações Globalstar, ICO e Iridium $\left(2^{\circ}\right.$, $3^{\circ}$ e $4^{\circ}$ gráficos, respectivamente e do alto para baixo).

Figura 4-7- Distribuição cumulativa complementar estimada para duração de eventos de desvanecimento, para o satélite um, da Constelação Globalstar, na região de Copacabana.

Figura 4-8 - Distribuição cumulativa complementar estimada para número de eventos de desvanecimento, para satélite mais elevado, da Constelação Globalstar. De cima para baixo: Copacabana, Irajá, Ipanema e Tijuca. 62

Figura 4-9 - Distribuição cumulativa complementar estimada para número de eventos de desvanecimento, para diversidade por combinação, da Constelação Globalstar. De cima para baixo: Copacabana, Irajá, Ipanema e Tijuca.

Figura 4-10 - Distribuição cumulativa complementar estimada para número de eventos de desvanecimento, para diversidade por comutação, da Constelação Globalstar. De cima para baixo: Copacabana, Irajá, Ipanema e Tijuca.

64

Figura 4-11 - Distribuição cumulativa complementar estimada para número de eventos de desvanecimento, para satélite mais elevado, da Constelação ICO. De cima para baixo: Copacabana, Irajá, Ipanema e Tijuca.

Figura 4-12 - Distribuição cumulativa complementar estimada para número de eventos de desvanecimento, para diversidade por combinação, da Constelação ICO. De cima para baixo: Copacabana, Irajá, Ipanema e Tijuca.

Figura 4-13 - Distribuição cumulativa complementar estimada para número de eventos de desvanecimento, para diversidade por comutação, da Constelação ICO. De cima para baixo: Copacabana, Irajá, Ipanema e Tijuca.

Figura 4-14 - Distribuição cumulativa complementar estimada para número de eventos de desvanecimento, para satélite mais elevado, da Constelação Iridium. De cima para baixo: Copacabana, Irajá, Ipanema e Tijuca.

Figura 4-15 - Distribuição cumulativa complementar estimada para número de eventos de desvanecimento, para diversidade por combinação, da 
Constelação Iridium. De cima para baixo: Copacabana, Irajá, Ipanema e Tijuca.

Figura 4-16 - Distribuição cumulativa complementar estimada para número de eventos de desvanecimento, para diversidade por comutação, da Constelação Iridium. De cima para baixo: Copacabana, Irajá, Ipanema e Tijuca.

Figura 4-17 - Distribuição cumulativa complementar estimada para duração de eventos de desvanecimento, para satélite mais elevado, da Constelação Globalstar. De cima para baixo: Copacabana, Irajá, Ipanema e Tijuca. 72

Figura 4-18 - Distribuição cumulativa complementar estimada para duração de eventos de desvanecimento, para diversidade por combinação, da Constelação Globalstar. De cima para baixo: Copacabana, Irajá, Ipanema e Tijuca.

Figura 4-19 - Distribuição cumulativa complementar estimada para duração de eventos de desvanecimento, para diversidade por comutação, da Constelação Globalstar. De cima para baixo: Copacabana, Irajá, Ipanema e Tijuca.

Figura 4-20 - Distribuição cumulativa complementar estimada para duração de eventos de desvanecimento, para o satélite mais elevado, da Constelação ICO. De cima para baixo: Copacabana, Irajá, Ipanema e Tijuca. 75

Figura 4-21 - Distribuição cumulativa complementar estimada para duração de eventos de desvanecimento, para diversidade por combinação, da Constelação ICO. De cima para baixo: Copacabana, Irajá, Ipanema e Tijuca.

Figura 4-22 - Distribuição cumulativa complementar estimada para duração de eventos de desvanecimento, para diversidade por comutação, da Constelação ICO. De cima para baixo: Copacabana, Irajá, Ipanema e Tijuca.

Figura 4-23 - Distribuição cumulativa complementar estimada para duração de eventos de desvanecimento, para o satélite mais elevado, da Constelação Iridium. De cima para baixo: Copacabana, Irajá, Ipanema e Tijuca. 78

Figura 4-24 - Distribuição cumulativa complementar estimada para duração de eventos de desvanecimento, para diversidade por combinação, da Constelação Iridium. De cima para baixo: Copacabana, Irajá, Ipanema e Tijuca.

Figura 4-25 - Distribuição cumulativa complementar estimada para duração de 
eventos de desvanecimento, para diversidade por comutação, da Constelação Iridium. De cima para baixo: Copacabana, Irajá, Ipanema e Tijuca.

Figura 4-26 - Distribuição cumulativa complementar estimada para número de inter-eventos de desvanecimento, para o satélite mais elevado, da Constelação Globalstar. De cima para baixo: Copacabana, Irajá, Ipanema e Tijuca.

Figura 4-27 - Distribuição cumulativa complementar estimada para número de inter-eventos de desvanecimento, para diversidade por combinação, da Constelação Globalstar. De cima para baixo: Copacabana, Irajá, Ipanema e Tijuca.

Figura 4-28 - Distribuição cumulativa complementar estimada para número de inter-eventos de desvanecimento, para diversidade por comutação, da Constelação Globalstar. De cima para baixo: Copacabana, Irajá, Ipanema e Tijuca.

Figura 4-29 - Distribuição cumulativa complementar estimada para número de inter-eventos de desvanecimento, para o satélite mais elevado, da Constelação ICO. De cima para baixo: Copacabana, Irajá, Ipanema e Tijuca.

Figura 4-30 - Distribuição cumulativa complementar estimada para número de inter-eventos de desvanecimento, para diversidade por combinação, da Constelação ICO. De cima para baixo: Copacabana, Irajá, Ipanema e Tijuca.

Figura 4-31 - Distribuição cumulativa complementar estimada para número de inter-eventos de desvanecimento, para diversidade por comutação, da Constelação ICO. De cima para baixo: Copacabana, Irajá, Ipanema e Tijuca.

Figura 4-32 - Distribuição cumulativa complementar estimada para número de inter-eventos de desvanecimento, para o satélite mais elevado, da Constelação Iridium. De cima para baixo: Copacabana, Irajá, Ipanema e Tijuca.

Figura 4-33 - Distribuição cumulativa complementar estimada para número de inter-eventos de desvanecimento, para diversidade por combinação, da Constelação Iridium. De cima para baixo: Copacabana, Irajá, Ipanema e Tijuca.

Figura 4-34- Distribuição cumulativa complementar estimada para número de 
inter-eventos de desvanecimento, para diversidade por comutação, da Constelação Iridium. De cima para baixo: Copacabana, Irajá, Ipanema e Tijuca.

Figura 4-35 - Distribuição cumulativa complementar estimada para duração de inter-eventos de desvanecimento, para satélite mais elevado, da Constelação Globalstar. De cima para baixo: Copacabana, Irajá, Ipanema e Tijuca.

Figura 4-36 - Distribuição cumulativa complementar estimada para duração de inter-eventos de desvanecimento, para diversidade por combinação, da Constelação Globalstar. De cima para baixo: Copacabana, Irajá, Ipanema e Tijuca.

Figura 4-37 - Distribuição cumulativa complementar estimada para duração de inter-eventos de desvanecimento, para diversidade por comutação, da Constelação Globalstar. De cima para baixo: Copacabana, Irajá, Ipanema e Tijuca.

Figura 4-38 - Distribuição cumulativa complementar estimada para duração de inter-eventos de desvanecimento, para satélite mais elevado, da Constelação ICO. De cima para baixo: Copacabana, Irajá, Ipanema e Tijuca.

Figura 4-39 - Distribuição cumulativa complementar estimada para duração de inter-eventos de desvanecimento, para diversidade por combinação, da Constelação ICO. De cima para baixo: Copacabana, Irajá, Ipanema e Tijuca.

Figura 4-40 - Distribuição cumulativa complementar estimada para duração de inter-eventos de desvanecimento, para diversidade por comutação, da Constelação ICO. De cima para baixo: Copacabana, Irajá, Ipanema e Tijuca.

Figura 4-41 - Distribuição cumulativa complementar estimada para duração de inter-eventos de desvanecimento, para satélite mais elevado, da Constelação Iridium. De cima para baixo: Copacabana, Irajá, Ipanema e Tijuca.

Figura 4-42 - Distribuição cumulativa complementar estimada para duração de inter-eventos de desvanecimento, para diversidade por combinação, da Constelação Iridium. De cima para baixo: Copacabana, Irajá, Ipanema e Tijuca.

Figura 4-43 - Distribuição cumulativa complementar estimada para duração de 
inter-eventos de desvanecimento, para diversidade por comutação, da Constelação Iridium. De cima para baixo: Copacabana, Irajá, Ipanema e Tijuca. 


\section{Lista de tabelas}

Tabela 2-1 - Combinações de estado de percurso para esquemas de diversidade de satélite usando até 4 satélites simultaneamente. 25

Tabela 2 - Definição dos processos aleatórios A (duração de eventos) e B (número de eventos), onde $D, P_{A}$ e $P_{B}$ representam respectivamente: duração do desvanecimento, peso estatístico atribuído a $\mathrm{A}$ e peso estatístico atribuído a B (reproduzida da referência [14]). 42

Tabela 4-1 - Número de observadores e prédios para cada região simulada. 46

Tabela 4-2 - Dados a respeito de órbitas e geometrias das constelações de satélites em estudo. 